\title{
CALCULATIONS OF LIGHT SCATTERING MATRIX OF DUST AEROSOLS FOR PROBLEMS OF LIDAR REMOTE SENSING
}

\author{
Natalia Kustova*,, Alexander Konoshonkin ${ }^{1}$, Anatoli Borovoi ${ }^{1}$, Zhenzhu Wang ${ }^{2}$, Dong Liu ${ }^{2}$, \\ Chenbo $\mathrm{Xie}^{2}$, Alexandra Tsekeri ${ }^{3}$, Josef Gasteiger ${ }^{4}$
}

${ }^{1}$ V.E. Zuev Institute of Atmospheric Optics SB RAS, Tomsk 634055, Russia

${ }^{2}$ Anhui Institute of Optics and Fine Mechanics, Chinese Academy of Sciences, Hefei 230031, China

${ }^{3}$ Institute for Astronomy Astrophysics Space Applications and Remote Sensing, Athens 15236, Greece ${ }^{4}$ Faculty of Physics, University of Vienna, Vienna 1010, Austria

*Email:kustova@iao.ru

\begin{abstract}
The light scattering matrix is calculated for large dust particles with irregular shape and refractive index of $1.3116+i 0.0$. The scattering matrix in the backward direction needed for lidar studies is separately discussed. In this case, the obtained results for the lidar and depolarization ratios are in good agreement with experimental data. It is shown that for randomly oriented particles the number of particle orientations needed for numerical calculations by exact methods like DDA becomes a crucial parameter. In particular, for particles with size parameters larger than 40 the number of orientations should be more than 1000 .
\end{abstract}

\section{INTRODUCTION}

Dust aerosols are of great importance for the Earth radiative balance. The optical characteristics of dust particles with realistic, irregular shapes can be calculated with good accuracy by exact methods like the finite-difference time-domain FDTD [1], the discrete dipole approximation (DDA) [2,3], and so on. However in literature there are rather few data for the scattering properties of irregular dust particles with size parameters larger than $20[4,5]$. The aim of this contribution is to fill up this gap using the physical-optics method (PO) [6]. Although the scattering properties shown herein do not represent the scattering properties of realistic dust particles considering the low refractive index, this is a first step towards the application of PO for dust particles.

\section{NUMERICAL RESULTS}

The optical properties of a particle at a given wavelength $\lambda$ depend on its microphysical characteristics such as shape, size, and the refractive index $m$. Size of nonspherical particles can be determined in different ways. We define the size parameter $x$ at a given wavelength $\lambda$ for a convex randomly oriented particle as

$$
x=2 \pi r / \lambda, r=\sqrt{C_{\text {geo }} / \pi},
$$

where $r$ is the radius of a sphere with equal geometric cross section and $C_{g e o}$ is the orientationaveraged geometrical cross-sectional area.

For numerical solutions of light scattering by randomly oriented particles of complex shapes, it is important to choose the necessary number of orientations [7]. Usually a criterion of the necessary number of orientations is some concurrence of the calculated and theoretical data for the extinction cross-section. Of course, such a criterion is not applicable to the lidar investigations where only the backscatter crosssection is measured. In this contribution, we state that the number of necessary orientations for the backscatter should be much more than for the concurrence of the extinction cross-section.

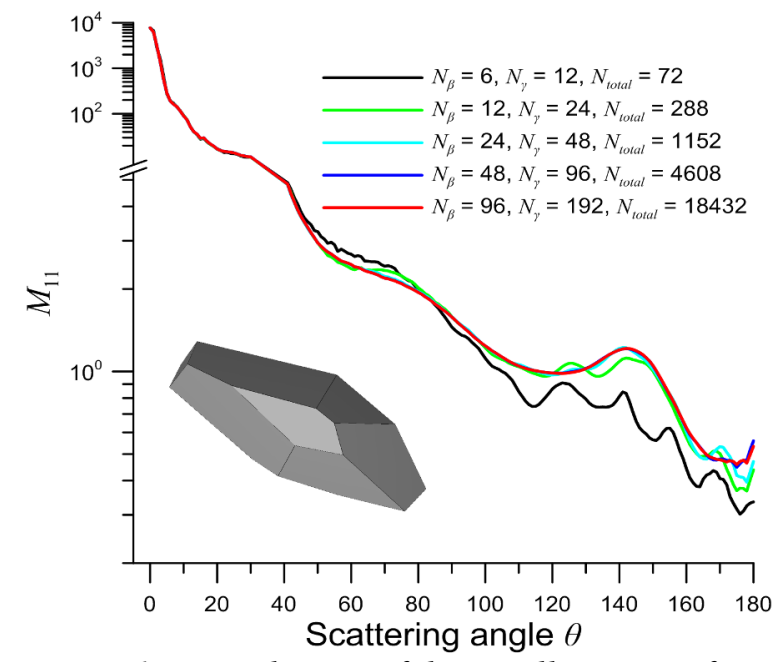

Figure 1. First element of the Mueller matrix for a randomly oriented dust particle calculated with the physical-optics approximation for different number of particle orientations. 
Figure 1 supports this statement. Here the first element of the Mueller matrix is presented for a typical shape of dust particles at $x=44.21, \lambda=$ $0.532 \mu \mathrm{m}$, and $m=1.3116+i 0.0$.

Figure 1 shows that the numerical data obtained for different orientation numbers oscillate more strongly in the backward scattering direction semi-sphere than in the forward counterpart. In particular, the numerical data converge more or less satisfactorily at 18432 orientations in the back hemisphere and at 72 orientations in the forward hemisphere. Since the forward scattering direction determines the extinction cross section according to the optical theorem, we guess that 72 orientations would be enough for the corresponding convergence of the extinction cross-section while this is not applicable to the backscatter. The same convergence is illustrated in Fig. 2 for other elements of the Mueller matrix.
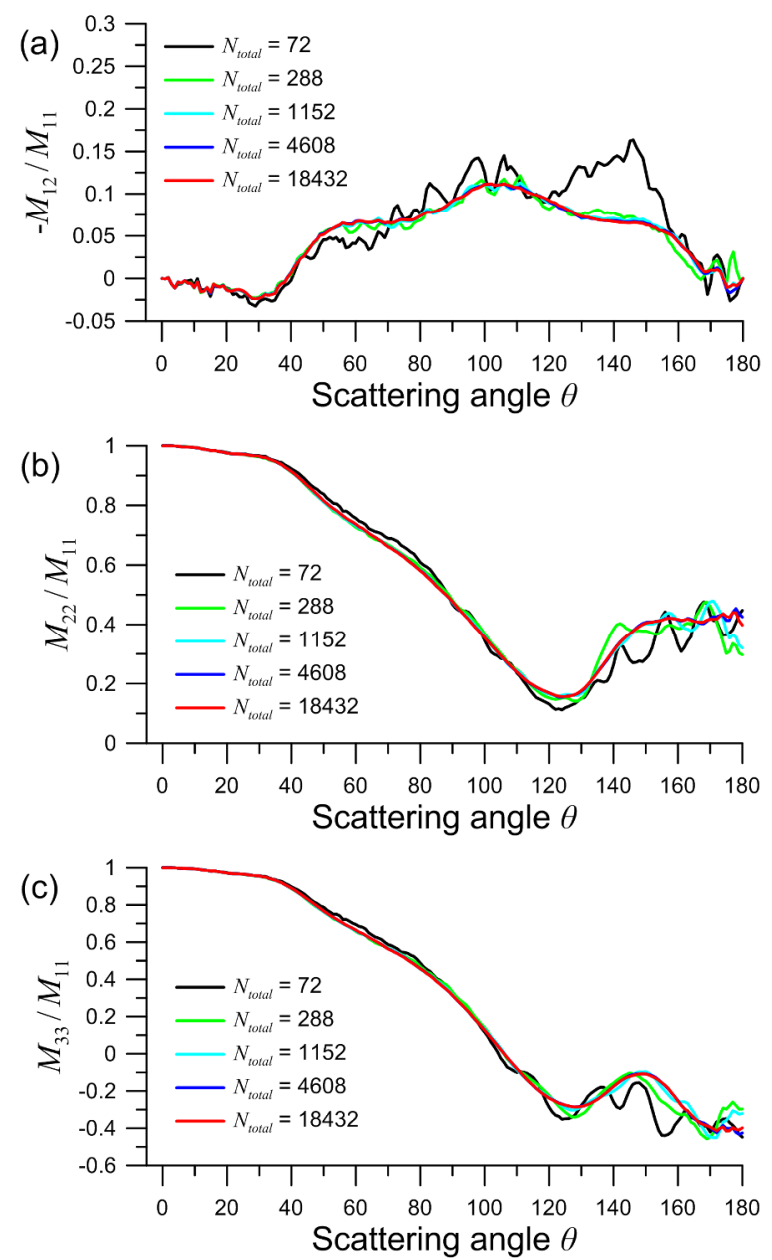
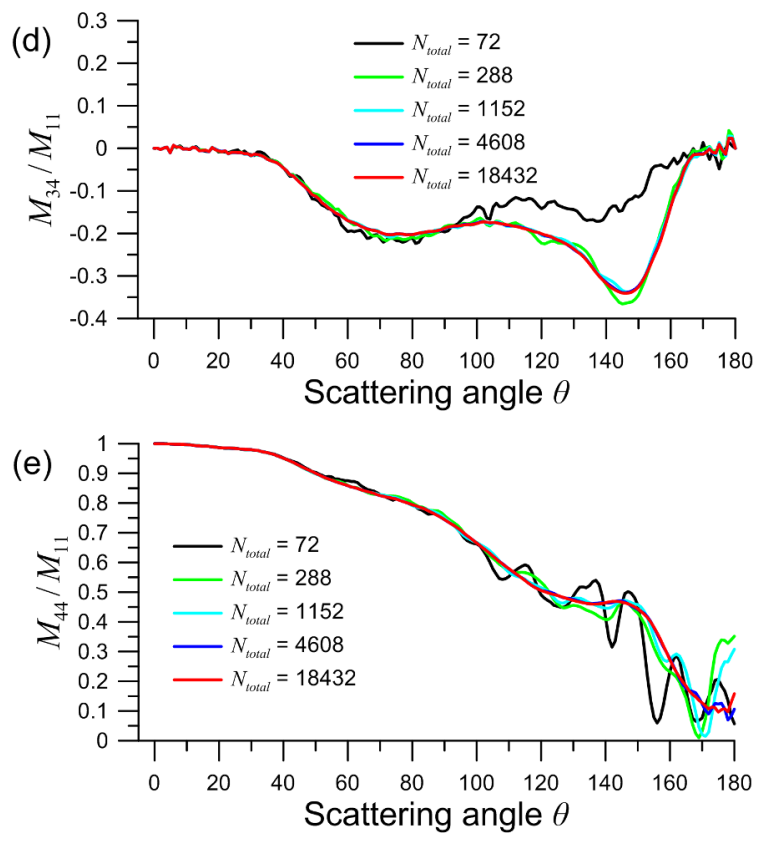

Figure 2. The same as in Fig. 1 for other elements of the Mueller matrix

This conclusion is easily explained within the framework of the physical-optics approximation as following. Let us determine a particle orientation by three Euler angles $\alpha, \beta$, and $\gamma$, where $\alpha$ describes particle rotation around the light incident direction, $\gamma$ describes rotation about an axis chosen inside a particle and $\beta$ corresponds to a tilt between the incident direction and the chosen axis. It is instructive to exclude the averaging over the angle $\alpha$ and to represent the Mueller matrix averaged only over the angles $\beta$ and $\gamma$. In this case, unlike Figs. 1 and 2, the Mueller matrix is represented as function of two variables: azimuth $\varphi$ and zenith $\theta$ scattering angles. Such two-dimensional functions are shown in Figs. 3 and 4 for different numbers of particle orientations.

Let us note that the averaging over angle $\alpha$ in our numerical algorithm is worked out analytically and this averaging is not included in the number of orientations. Figures 3 and 4 demonstrate that the interrupted view of the two-dimensional functions is created by the discrete sets of the orientations used at the numerical averaging. These interrupted views of the functions prove that the number of orientations was not sufficient for the reliable averaging over the random 
orientations. And only at larger number of orientations the Mueller matrixes become smooth functions demonstrating that the orientation number was more or less satisfactory.
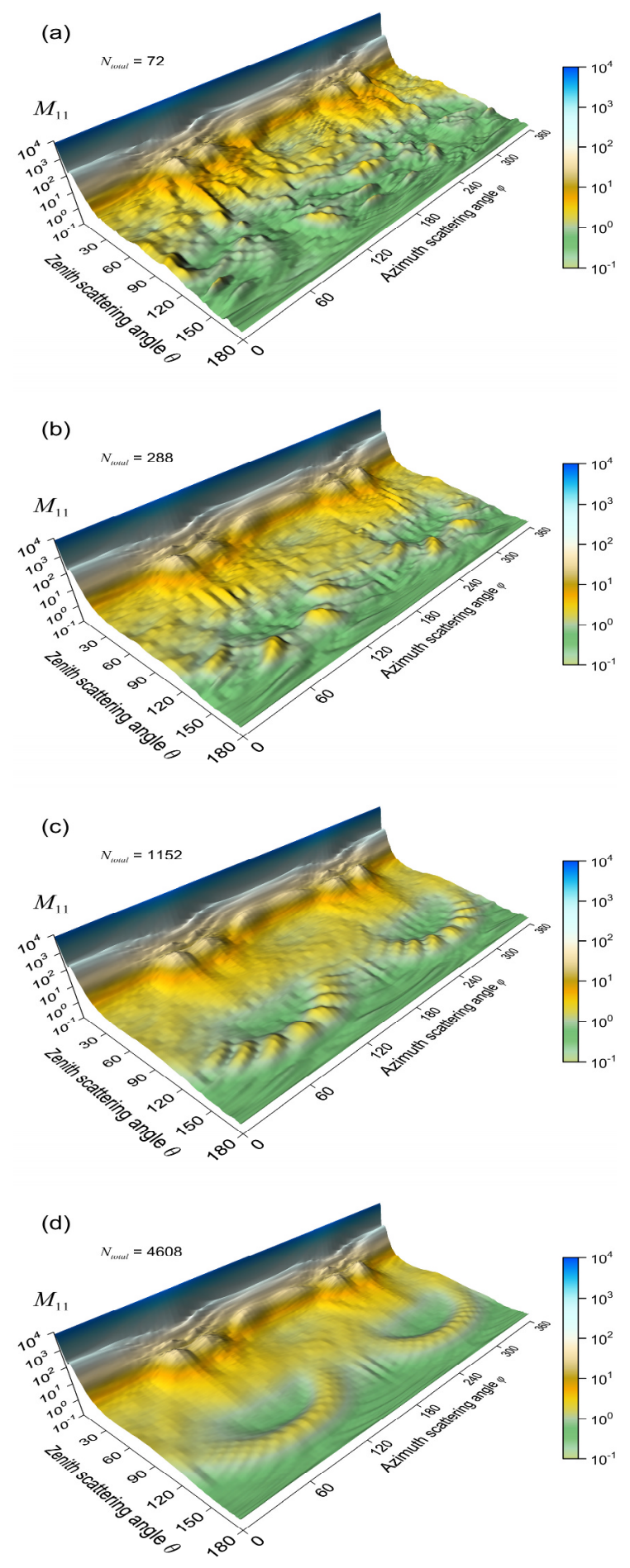

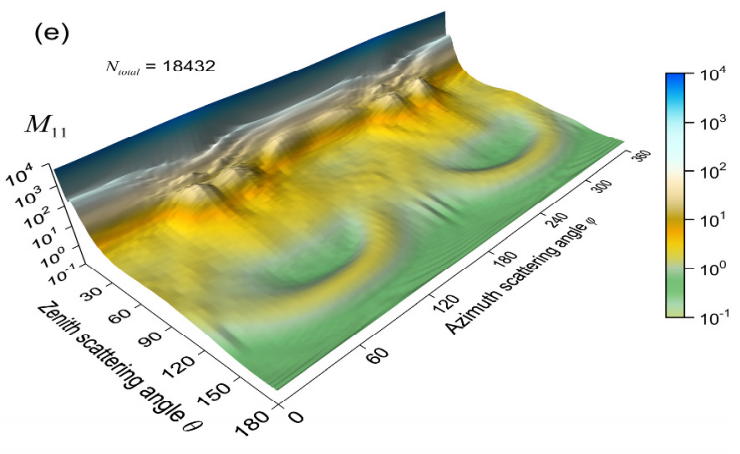

Figure 3. The first element of the Mueller matrix at the partial averaging over particle orientations for different numbers of orientation.
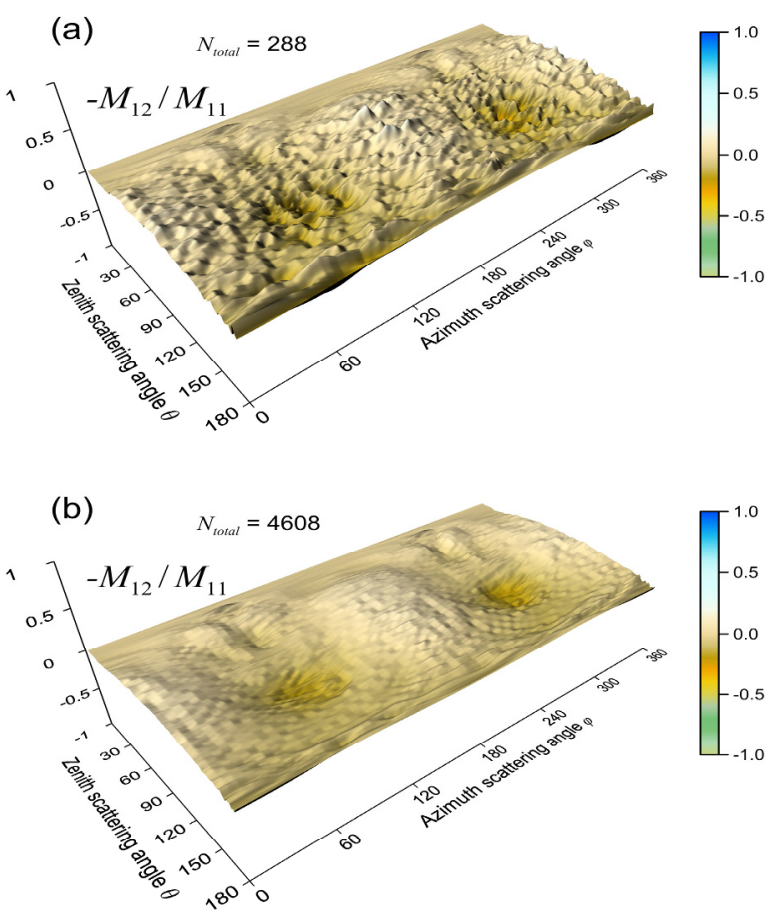

Figure 4. The same as in Fig. 3 for the element $M_{12}$

In the forward scattering direction, the diffracted field that is weakly changing with particle orientations becomes predominant. Similarly, the light beams scattered near the forward direction undergo a little number of reflections inside the faceted particles.

Therefore the scattering to the forward hemisphere is less sensitive to the orientation numbers as compared with the backward hemisphere. 
The simple estimation of the necessary orientation numbers can be described by the equation

$$
N_{\text {total }}=N_{\beta} \cdot N_{\gamma} \cdot N_{\alpha}=\frac{\pi}{0.3 \xi} \cdot \frac{2 \pi}{0.3 \xi} \cdot \frac{2 \pi}{0.3 \xi},
$$

where $\xi=\lambda / a$ is the diffraction angle, $a-$ the maximum size of a side of a faceted particle. In Eq. (2), the rotation angle of a particle is taken as $1 / 3$ of the diffraction angle. This is the upper estimate. In practice, anybody may decrease the estimate in 2-3 times. Such an estimate corresponds to our calculations presented in Fig. 1. Indeed, the convergence should be at $167 \cdot 335=55945$ orientations according to Eq. (2) while the convergence takes place already at 18432 orientations.

(a)

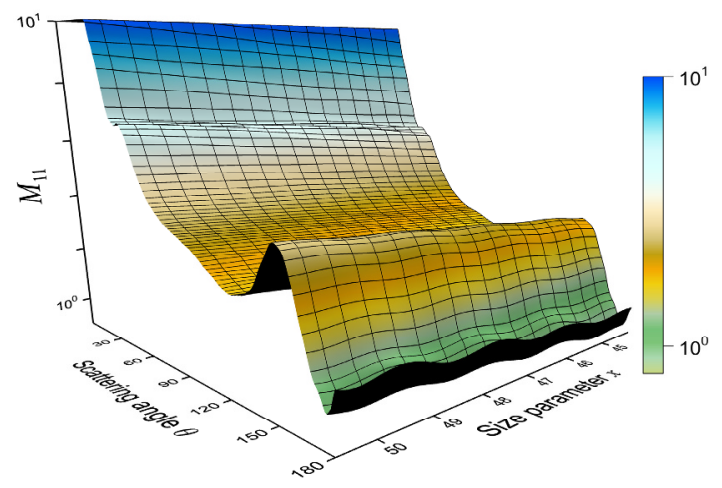

(b)

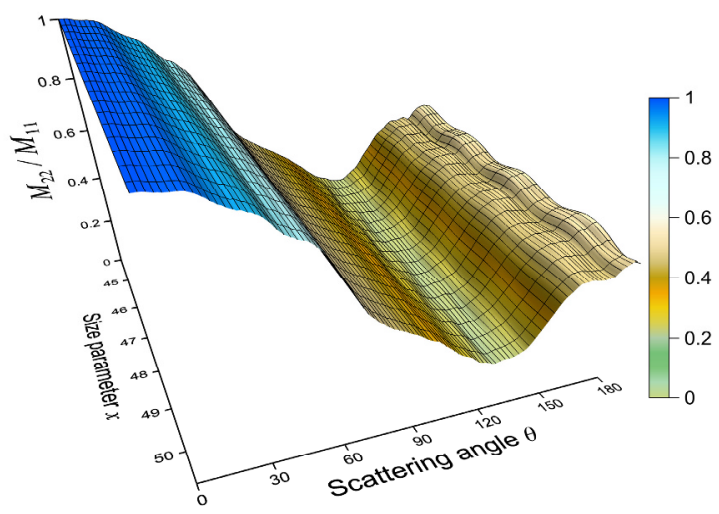

Figure 5. Interference oscillations for the backscattering Mueller matrix

It is worthwhile to note that a change of the particle size leads to interference oscillations at the backward scattering direction which are important in lidar studies. Such oscillations are seen both for $M_{11}$ and $M_{22}$ elements (see Fig. 5). Here the maximum quantity is transformed to the minimum quantity if a change of the size parameter is equal to one. Consequently, correct calculations of the backscattering Mueller matrix should be done with the mesh less than one for the size parameter.

\section{CONCLUSION}

Thus, in calculations of the light scattering matrixes for large particles with irregular shape resembling dust particles, but having low refractive index of $1.3+\mathrm{i} 0$ (size parameter more than 40) it is necessary to take more than 1000 particle orientations.

Number of the necessary orientations increases essentially with particle size. Moreover, the step of calculations relative to the size parameter should be less than one. This circumstance also increases the calculation cost. Such facts are usually underestimated in the literature. As a result, the exact methods like DDA should meet a lot of difficulties while our physical-optics approximation looks as a promising method for solving this problem.

\section{ACKNOWLEDGEMENTS}

This work is supported by the RFBR under Grants no. 18-05-00568, no. 18-55-53046, and budget project AAAA-A17-117021310145-6. Calculation of Mueller matrixes is supported only by Russian Science Foundation (18-77-10035).

\section{REFERENCES}

[1] H. Ishimoto, Y. Zaizen, A. Uchiyama, K. Masuda, Y. Mano, J. Quant. Spectrosc. Radiat. Transfer 111, 2434-2443 (2010)

[2]T. Mehri, O. Kemppinen, G. David, H. Lindqvist, J. Tyynelä, T. Nousiainen, P. Rairoux, A. Miffre, J. Atmos. Res. 203, 44-61 (2018)

[3] M. A. Yurkin, V. P. Maltsev, A. G. Hoekstra， J. Quant. Spectrosc. Radiat. Transfer 106, 546-557 (2007)

[4] J. Gasteiger, $\quad$ M. Wiegner, S. Groß, V. Freudenthaler, C. Toledano, M. Tesche, K. Kandler, Tellus B: Chemical and Physical Meteorology 63(4), 725-741 (2011)

[5] Y. Grynko, Y. Shkuratov, J. Förstner Optics Letters 41, 3491-3494 (2016).

[6]A. Borovoi, A. Konoshonkin, N. Kustova, J. Quant. Spectrosc. Radiat. Transfer 146, 181-189 (2014)

[7] A. V. Konoshonkin, A. G. Borovoi, N. V. Kustova, H. Okamoto, H. Ishimoto, Y. Grynko, J. Förstner, J. Quant. Spectrosc. Radiat. Transfer 195, 132-140 (2017) 